\title{
A análise do uso da técnica Delphi na tomada de decisão em pacientes críticos: uma revisão sistemática
}

\author{
Analysis of Delphi technique for decision making in critical ill patients:
}

\author{
a systematic review
}

\section{Ana Paula Freitas Bahia dos Santos ${ }^{1}$, Jessika de Freitas Andrade ${ }^{1}$, Geisa Cristina da Silva Alves $^{2}$, Samuel Dutra da Silva ${ }^{3}$, Cristina Sanches ${ }^{4}$, Farah Maria Drumond Chequer ${ }^{4}$}

Santos APFB, Andrade JF, Alves GCS. Silva SD, Sanches C. Chequer FMD. A análise do uso da técnica Delphi na tomada de decisão em pacientes críticos: uma revisão sistemática / Analysis of the use of the Delphi technique for decision-making in critically ill patients: a systematic review. Rev Med (São Paulo). 2020 maio-jun.;99(3):291-304.

RESUMO: Introdução: A técnica Delphi é um método utilizado para alcançar consenso entre especialistas sobre determinado assunto. Objetivos: realizar revisão sistemática sobre a contribuição e utilização do método Delphi exclusivamente por médicos, para tomada de decisões em pacientes críticos. Métodos: A pesquisa foi realizada nas bases de dados: PubMed, SciElo, Biblioteca Virtual em Saúde, Scopus, Periódicos CAPES e ClinicalTrials.gov, utilizando os descritores: "Delphi technique", "Decision making", "Critical care" e "Physicians". Selecionou-se ensaios clínicos ou estudos observacionais para responder à pergunta norteadora: "Qual a contribuição da técnica Delphi na tomada de decisão pelos médicos em pacientes críticos?" Para avaliação do nível de evidência e grau de recomendação dos estudos incluídos, foi utilizado a escala do Centro de Medicina Baseada em Evidências de Oxford. Resultados: Foram incluídos dezoito referências para compor este estudo. As contribuições do método Delphi foram consideradas importantes para a tomada de decisões em quinze estudos analisados, para: definir estratégias de manejo, tratamento e recomendações; gerenciar e diagnosticar pacientes; definir consenso para exames; identificar ideias controversas e seus motivos subjacentes. Das limitações do método, a mais recorrente foi a seleção de especialistas de diferentes níveis de conhecimento. Conclusão: Este estudo mostra a aplicabilidade da técnica Delphi para manejo de pacientes críticos, tratamento medicamentoso, recomendações para sintomas específicos, diretrizes para gerenciar e diagnosticar pacientes, consenso para testes e exames e identificação de tópicos de ideias controversas e seus motivos subjacentes em situações onde não há consenso na literatura médica.

Descritores: Técnica Delfos; Tomada de decisões; Cuidados críticos; Unidades de Terapia Intensiva.
ABSTRAT: Introduction: The Delphi technique is a method used to reach consensus among experts on a given subject. Objectives: To develop a systematic review about the contribution and use of the Delphi method exclusively by physicians, for decision-making in critically ill patients. Methods: The study was conducted in the databases: PubMed, SciElo, Biblioteca Virtual em Saúde, Scopus, CAPES Periodicals and ClinicalTrials.gov, using the terms: "Delphi technique", "Decision making", "Critical care" and "Physicians". Clinical trials or observational studies were selected to answer the guiding question: "What is the contribution of the Delphi technique to decision-making by physicians in critically ill patients?" To evaluate the level of evidence and degree of recommendation for the included studies, the scale from the Center for Evidence-Based Medicine in Oxford was used. Results: Eighteen articles were included to compose this study. The contributions of the Delphi method were considered important for the decision-making in fifteen of the studies analyzed, to: define management strategies, treatment and recommendations; manage and diagnose patients; set consensus for examinations; and identify controversial ideas and their underlying motives. From the limitations of the method, most frequent was the selection of specialists from different levels of knowledge. Conclusion: This study shows the applicability of the Delphi technique for critical patient management, drug treatment, recommendations for specific symptoms, guidelines for managing and diagnosing patients, consensus for tests and exams, and identification of topics of controversial ideas and their underlying motives in situations where there is no consensus in the medical literature.

Keywords: Delphi technique; Decision making; Critical care; Physician; Intensive Care Units.

Apresentado: IX Jornada Acadêmica de Medicina da Universidade de Itaúna, Itaúna, MG, 9 nov. 2017.

1. Universidade de Itaúna, Itaúna, MG. Acadêmicas de medicina. ORCID: Santos APFB - https://orcid.org/0000-0001-6720-5367; Andrade JF - https:// orcid.org/0000-0002-2868-0883. Email: anapaulafreitasb@gmail.com, jessikafreitas08@gmail.com

2. Universidade Federal de São João del-Rei, Campus Centro-Oeste Dona Lindu (UFSJ-CCO), Divinópolis, MG. Enfermeira - Doutoranda em Ciências da Saúde. ORCID: https://orcid.org/0000-0002-2023-8011.Email:_geisa.cristina@gmail.com

3. Universidade de Itaúna, Itaúna, MG. Médico, preceptor de medicina e mestre pela Universidade Federal de São João del-Rei, Campus Centro-Oeste Dona Lindu (UFSJ-CCO), Divinópolis, MG. ORCID: https://orcid.org/0000-0002-4287-3542. Email: samueldutradasilva@gmail.com

4. Universidade Federal de São João del-Rei, Campus Centro-Oeste Dona Lindu (UFSJ-CCO), Divinópolis, MG. Farmacêutica - Professora Adjunto. ORCID: Sanches C - https://orcid.org/0000-0002-8562-1337; Chequer FMD - https://orcid.org/0000-0003-3514-2132. Email:_csanches@ufsj.edu. br, farahchequer@ufsj.edu.br.

Endereço para correspondencia: Farah Maria Drumond Chequer. Universidade Federal de São João del-Rei, Campus Centro-Oeste Dona Lindu (UFSJ-CCO). Rua Sebastião Gonçalves Coelho 400, Chanadour. Divinópolis, MG. CEP: 35.501-296. E-mail: farahchequer@ufsj.edu.br. 


\section{INTRODUÇÃO}

A técnica Delphi foi desenvolvida por Dalkey

Ae Helmer, na Rand Corporation na década de 1950 e usada, primariamente, para obter opiniões de especialistas sobre um projeto de defesa militar ${ }^{1}$. É um método amplamente utilizado e aceito para alcançar consenso entre especialistas sobre determinado assunto ${ }^{2}$. É adequado para o desenvolvimento de critérios de diagnóstico na ausência de um padrão-ouro e é um método reconhecido aceito e usado para reunir informações de especialistas dentro de um domínio de proficiência ${ }^{3,4}$.

A técnica Delphi é feita com a participação de indivíduos que possuem o conhecimento e experiência em determinada área. Essencialmente, não existe um número específico de participantes necessários para sua realização. O método se inicia a partir da distribuição de um questionário a respeito do assunto para cada especialista. Depois de reunir as opiniões destes, o resultado é compilado. O método é executado por meios de rodadas de questionários e feedback das opiniões dos participantes, a fim de se chegar a um consenso sobre determinado assunto. Deve-se estabelecer, previamente, qual a porcentagem necessária de aprovação pelos participantes para que uma resposta a determinada pergunta seja considerada como acordo. Caso um item não seja aceito pelo mínimo de profissionais, deve-se alterá-lo conforme sugestões dos participantes, a fim de se alcance tal aprovação. Após as rodadas necessárias para se chegar ao consenso dos itens sugeridos, estes serão classificados como aprovados ou não ${ }^{1}$.

Quanto às vantagens da utilização da técnica Delphi, é possível citar: permite incluir vários especialistas de diversas áreas e regiões geográficas, sem a necessidade de encontrar-se pessoalmente; os participantes ficam anônimos, eliminando a influência de pessoas influentes no grupo ${ }^{4-5-6}$. É efetivo por promover reflexão e elaborar diretrizes $^{6}$; economiza tempo e custo pelo fato de não necessitar encontros pessoais ${ }^{7}$.

A técnica Delphi trata-se de uma ferramenta fundamental em temas onde ainda existem controvérsias na literatura ${ }^{3,4}$ e pode ser usada para definir cuidados clínicos adequados $^{8}$. Isso justifica estudos como este que aborde de forma clara e direta a efetividade dessa técnica na medicina, que apesar de ser um campo onde o método tem grande potencial de atuação é crucial a avaliação da sua eficácia e viabilidade para se chegar a um consenso no cotidiano médico.

Portanto, este artigo teve como objetivo desenvolver uma revisão sistemática sobre a contribuição do uso do método Delphi para tomada de decisões exclusivamente pelos médicos em pacientes críticos, internados em CTI (Centro de Tratamento Intensivo) /UTI (Unidade de Tratamento Intensivo). Foi escolhido este cenário (pacientes críticos) devido à necessidade da urgência na tomada de decisão pelos médicos sendo que, geralmente, podem ocorrer dúvidas sobre quais intervenções e/ou diagnósticos a serem realizados pelos médicos.

\section{MÉTODOS}

Buscando analisar a aplicabilidade da técnica Delphi, foi desenvolvida uma revisão sistemática de artigos já publicados com informações acerca do assunto.

Desenho do estudo: Para a elaboração da revisão sistemática utilizou-se o Guideline The PRISMA Statement ${ }^{9}$.

Pergunta norteadora da pesquisa: "Qual a contribuição da técnica Delphi para tomada de decisão pelos médicos em pacientes críticos?”.

Definição da População, Intervenção, Comparação, Desfechos e Estudos (PICOS): De acordo com a pergunta norteadora foi estabelecido o "PICOS""10: "P" (população): Uso da técnica Delphi pelos médicos; "I" (intervenção): uso do método Delphi; "C" (comparação): não tem comparação; "O”" (desfechos): efetividade da aplicação da técnica Delphi no caso de pacientes críticos; "S" (tipos de estudos): estudos observacionais e clínicos.

Fontes de informação: A busca dos estudos para compor esta revisão foi realizada nas bases de dados: PubMed, SciELO, Biblioteca Virtual em Saúde (BVS), Periódicos CAPES e Scopus. Também foram pesquisados registros de ensaios clínicos de estudos em andamento ou recentemente concluídos no ClinicalTrials.gov. O medical Subject Headings (MeSH) foi utilizado para definir os descritores a seguir: "Delphi technique", "Decision making", "Critical care" e "Physicians" utilizados em conjunto com o operador booleano "AND" nas bases de dados Pubmed, SciELO, Biblioteca Virtual em Saúde (BVS) e Periódicos CAPES, Scopus e ClinicalTrials. gov. Para a busca no Pubmed realizou-se também a busca utilizando os mesh term e seus entretermos: "Delphi Technique"; Delphi Techniques; Technique, Delphi; Techniques, Delphi; Delphi Technic; Delphi Technics; Technic, Delphi; Technics, Delphi; Delphi Studies; Delphi Study; Studies, Delphi; Study, Delphi; "Critical care"; Care, Critical; Intensive Care; Care, Intensive; Surgical Intensive Care; Care, Surgical Intensive; Intensive Care, Surgical, "Decision making"; Decision Making, Shared; Decision Makings, Shared; Making, Shared Decision; Makings, Shared Decision; Shared Decision Making; Shared Decision Makings, "physicians".

Estudos obtidos de outras fontes: Também foram analisadas as referências dos artigos selecionados após a aplicação dos critérios de inclusão e exclusão.

Seleção dos estudos: Após a seleção dos artigos pela estratégia de busca em cada base de dados realizou-se um cruzamento dos artigos para retirada de publicações duplicadas.

Critérios de elegibilidade: Foram considerados elegíveis para esta revisão ensaios clínicos ou estudos 
observacionais realizados em humanos, publicados até junho de 2017, nos idiomas inglês, espanhol e português, e que descreveram sobre a aplicabilidade da técnica Delphi e seu uso por médicos a fim de auxiliar na tomada de decisão em pacientes críticos. Foram excluídos os artigos de revisão, os editoriais, as cartas ao editor, as notícias, os comentários e os resultados de dissertações, teses ou de resumos publicados em anais de congresso ou revista científica. A utilização da técnica Delphi por outros profissionais de saúde foi considerada como critério de exclusão. Esses critérios foram checados independentemente de maneira cega por dois autores para a inclusão e exclusão dos estudos encontrados. Em caso de discordância entre os revisores, outros membros da equipe foram acionados para gerar o consenso.

Avaliação do nível de evidência e análises adicionais: Para avaliação do nível de evidência e grau de recomendação dos estudos incluídos foi utilizado a escala do Centro de Medicina Baseada em Evidências de Oxford $^{11}$ que avalia cada estudo de acordo com sua metodologia, conforme mostra a Tabela 1 .
Para avaliar o grau de concordância entre os dois avaliadores (APFBS e JFA) foi utilizado o Coeficiente $\mathrm{Kappa}^{12}$. Este teste foi realizado com intervalos de confiança de 95\%, no programa Stata, versão 10.0.

Coleta e análise dos dados: Foi realizada a leitura na íntegra dos artigos que atenderam aos critérios de inclusão e, durante essa fase, foram coletadas as variáveis: autor/ ano/ localidade; objetivo do estudo; tamanho da amostra (número de médicos participantes); tempo do uso do método Delphi; descrição da contribuição do uso do método Delphi para tomada de decisões em pacientes críticos; efetividade; limitações; aplicabilidade da técnica.

\section{RESULTADOS}

Foram selecionados 33 estudos a partir da leitura dos resumos dos quais 15 foram excluídos por não cumprir os critérios de elegibilidade conforme descrito em Figura 1.

Apenas 18 estudos atendiam aos critérios de inclusão e foram elegíveis para esta revisão conforme Figura 2.

Tabela 1. Nível de Evidência Científica por Tipo de Estudo - "Oxford Centre for Evidence-based Medicine"

\begin{tabular}{|c|c|c|}
\hline Grau de recomendação & Nível de evidência & Tratamento - Prevenção - Etiologia \\
\hline $\begin{array}{l}\text { B } \\
\text { Estudo que recomenda a ação; são encontradas evidências } \\
\text { importantes no desfecho, e a conclusão é de que há benefício na } \\
\text { escolha da ação em relação aos riscos do dano. Há evidências } \\
\text { razoáveis para apoiar a recomendação }\end{array}$ & 2B & Decisão de análise \\
\hline $\begin{array}{l}\text { C } \\
\text { Consiste em estudos de nível } 4 \text { ou generalização de estudos } \\
\text { de nível } 2 \text { ou } 3 \text {. Encontra-se mínimas evidências satisfatórias } \\
\text { na análise dos desfechos, mas conclui que os benefícios e } \\
\text { os riscos do procedimento não justificam a generalização da } \\
\text { recomendação. Há evidências insuficientes, contra ou a favor }\end{array}$ & $2 \mathrm{C}$ & $\begin{array}{l}\text { Observação de resultados terapêuticos } \\
\text { (outcomes research). }\end{array}$ \\
\hline
\end{tabular}

Adaptado: http://www.cebm.net/index.aspx?o=1025. Fonte: Phillips et al. ${ }^{11}$

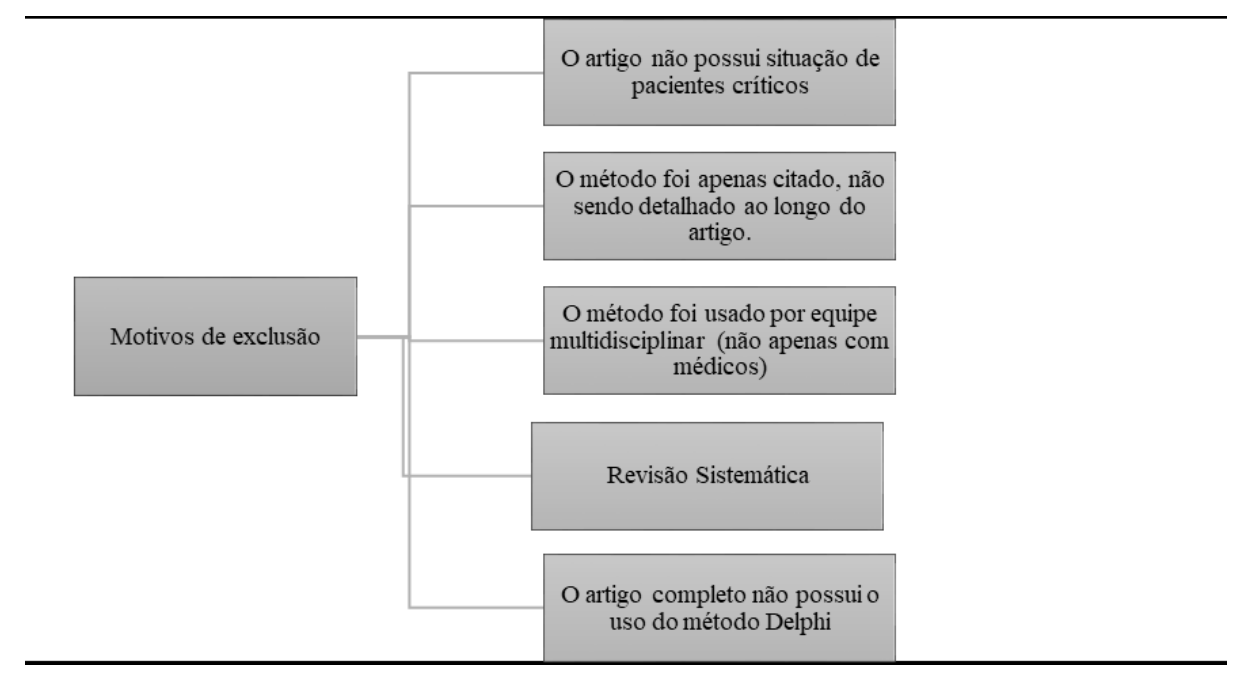

Figura 1: Motivos de destituição dos estudos por não se adequarem aos critérios de inclusão $(\mathrm{n}=15)$ 


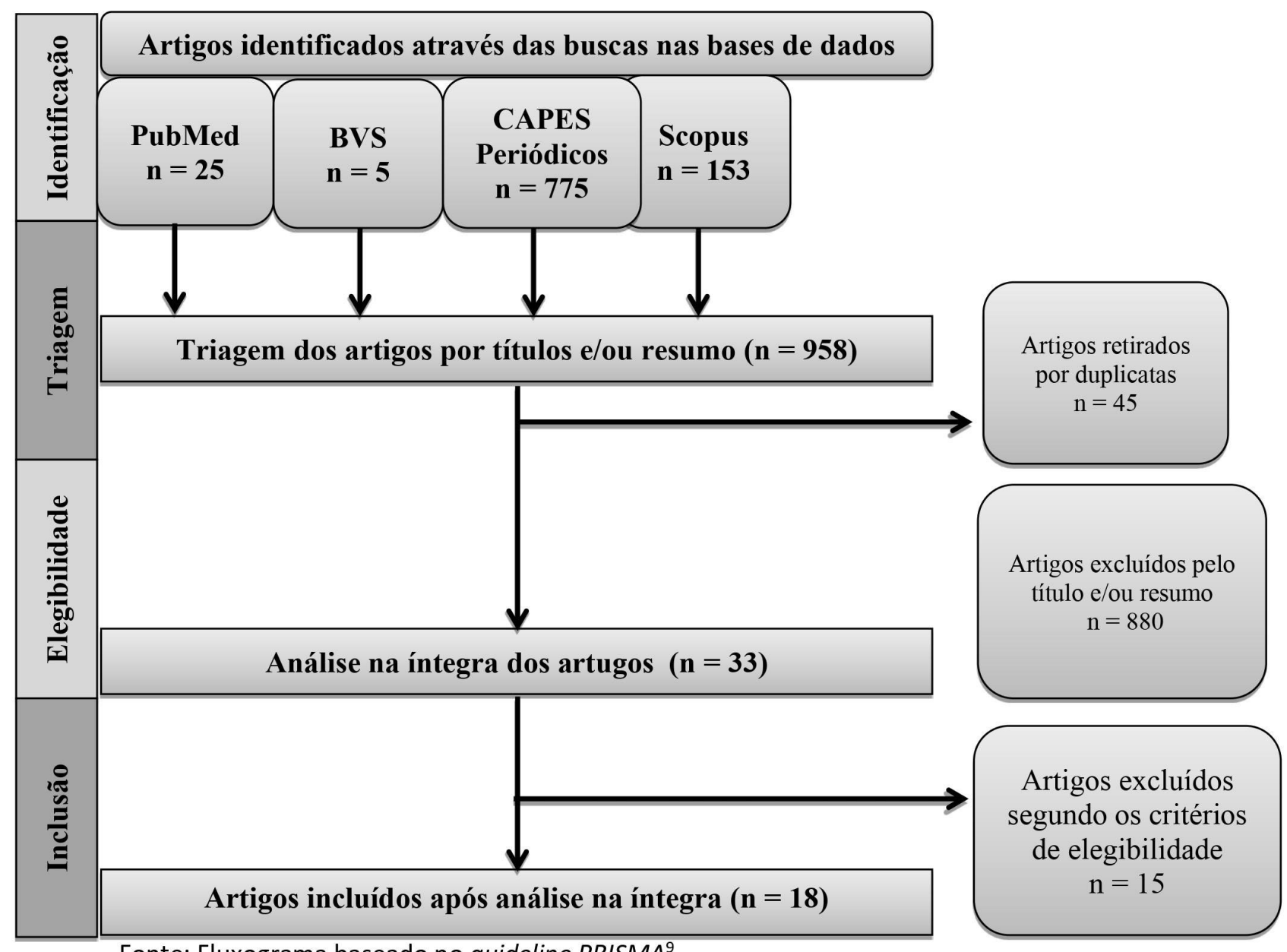

Fonte: Fluxograma baseado no guideline PRISMA ${ }^{9}$

Figura 2: Fluxograma das fases da revisão sistemática.

O grau de concordância entre os dois autores foi considerado como acordo substancial de kappa teste $=$ $0.7150^{12}$.

Dos 18 artigos selecionados segundo os critérios de elegibilidade, todos consistem em estudos observacionais realizados do ano de 2001 à 2016 em diferentes países sendo eles EUA' ${ }^{13-19}$, Reino Unido ${ }^{20,21}$, Canadáa ${ }^{3,22,23}$, Coreia do $\mathrm{Sul}^{2}$, Portugal ${ }^{5}$, Austrália $^{24}$, França ${ }^{7}$ e Espanha ${ }^{25,26}$.

Em relação ao nível de evidência e grau de recomendação dos estudos selecionados, quatorze (78\%) estudos foram classificados com evidência $2 \mathrm{c}$ e nível IV de recomendação, consiste em estudos com poucas evidências para análise dos desfechos, mas conclui que os benefícios e os riscos do procedimento não justificam a generalização da recomendação. Outros quatro estudos foram considerados com evidência $2 \mathrm{~b}$ e nível II de recomendação, com bons padrões de referências para decisões.

Em relação aos objetivos da técnica Delphi, em dois estudos o método foi utilizado para desenvolver listas sejam de verificação ${ }^{24}$ ou separação de by-pass $^{18}$. Em dois dos artigos, o método foi empregado no intuito de propor diretrizes para o manejo dos pacientes ${ }^{13,15}$. Outro artigo buscou estabelecer diretrizes para uso de ultrassonografia a beira do leito ${ }^{19}$. Em três estudos o método foi utilizado para desenvolver critérios de admissão na $\mathrm{UTI}^{7}$ e em casos de exacerbação da doença pulmonar obstrutiva crônica (DPOC) ${ }^{25,26}$. Três artigos tiveram a aplicação do método como fonte de consenso, seja para tomada de decisões apropriadas para o tratamento e diagnóstico ${ }^{14,16,20} \mathrm{ou}$ para avaliar a necessidade da consulta com o especialista e avaliação de testes e exames importantes na sala de emergência ${ }^{2}$ ou para desenvolver conformidade na prescrição de analgesia e sedação ${ }^{20}$. Em um estudo o método foi utilizado para avaliação da melhor técnica para ventilação mecânica ${ }^{21}$, enquanto outro artigo utilizou-se do método para desenvolver critérios para identificar os 
limiares em equipamentos de monitorização ${ }^{23}$. Três artigos apresentaram aplicações mais específicas como a análise de tópicos controversos entre anestesistas e obstetras ${ }^{3}$, a prática nos cuidados paliativos no fim da vida ${ }^{5}$ e práticas importantes nos pacientes com insuficiência respiratória ${ }^{17}$.

Do número de médicos participantes do método Delphi, em nove dos estudos analisados houve participação integral dos membros em todas as etapas ${ }^{2,3,7,15-17,19,25,26}$. Em outros oito artigos ocorreu uma perda dos participantes no decorrer das rodadas $5,13,14,18,20,21,23,25$, contudo, esse afastamento foi de aproximadamente $26,16 \%$ dos especialistas. Em um artigo cada rodada consistiu em dois questionários sendo o estudo composto por três rodadas. Houve variáveis no número de participantes em uma mesma rodada, entretanto a média de participação neste estudo foi de 10,66 participantes. O número de profissionais em cada estudo inicialmente variou de 10 a 79 participantes com uma média de 30,17 médicos convidados. Um dos artigos não citou o número de médicos participantes ${ }^{19}$.

Quanto ao tempo de uso do método Delphi, 15 dos 18 artigos selecionados não relataram a duração da aplicação do procedimento ${ }^{2,7,13,14,16-26}$. Em um artigo, o período de aplicabilidade foi de janeiro de 2006 a $2007^{15}$. Nos outros dois trabalhos, o período ficou entre fevereiro a abril de $2012^{5}$ e outubro de 2012 a janeiro de $2013^{3}$.

No que se refere às contribuições do método Delphi para a tomada de decisões dos profissionais médicos, em 14 dos estudos o uso da técnica foi importante para se chegar a definição de estratégias de manejo, tratamento medicamentoso, recomendações para sintomas específicos e uso de ultrassonografia, diretrizes para gerenciar e diagnosticar pacientes, assim como conseguiu consenso para testes e exames além de identificar tópicos de ideias controversas e seus motivos subjacentes e estabelecer determinantes no desfecho da DPOC 2,3,5,13-20,22-26. Em um dos trabalhos, o autor ressaltou a importância da técnica Delphi para promover um amplo movimento de reflexão e desafios para os profissionais ${ }^{5}$. Ademais, em três artigos o método não apresentou contribuição significativa $a^{7,21,24} \mathrm{em}$ um destes a lista de verificação formulada não apresentou diferença relevante do seu uso para o diagnóstico ${ }^{24}$, em outro estudo, o autor relatou que a ineficácia do método se deve a não descrição dos pensamentos e ideias que levam a opinião do profissional ${ }^{21}$, enquanto que um destes artigos relatou que os critérios desenvolvidos não foram pertinentes para aplicação ${ }^{7}$.

O resultado da efetividade da técnica Delphi foi avaliado pelos autores Elliot ${ }^{24}$, Baumann ${ }^{13}$, Lee $^{2}$, Mendes ${ }^{5}$, Ogden ${ }^{18}$ e Hawryluck ${ }^{22}$ através da Escala LIKERT, e pelos autores Dellinger ${ }^{15}$ e Frankel ${ }^{19}$ através do sistema GRADE. Já os autores Garroust ${ }^{7}$, Quintana ${ }^{26}$ e Garcia Gutierrez ${ }^{25}$ utilizou-se de uma tabela de admissão com variação entre 1 e 9, considerando intervalos entre estes valores que demonstraram níveis de concordância de acordo com a pontuação. Dentre os 18 artigos, cinco consideraram consenso quando a ideia era aceita por $80 \%$ ou mais dos participantes ${ }^{5,14,19,22,25}$. Em três artigos o consenso foi considerado maior ou igual a $70 \%$ de aceitação ${ }^{2,16}$. Em dois artigos o consenso foi atingido quando se obteve valores iguais ou maiores que $75 \%{ }^{17,23}$. Em outros três artigos, valores iguais ou maiores que $60 \%$ e $66 \%$ foram considerados consenso ${ }^{3,4,13,18,21}$. Em três artigos ${ }^{15,17,20,24}$ esses valores não foram citados, apenas se mencionou "um forte acordo" em um deles 7 . Seis entre os 18 trabalhos destacaram que as estratégias aprovadas pelo consenso foram selecionadas em mais de uma rodada ${ }^{5,16-18,20,23}$. Os outros 12 estudos ${ }^{2,3,7,13-15,19,21,22,24-26}$ não se referiram a esse episódio.

Dois dentre os 18 artigos não relatam as limitações encontradas no decorrer do uso da técnica Delphi ${ }^{5,15}$. Sete trabalhos ${ }^{2,3,7,13,14,18,20}$, citaram a seleção dos especialistas como uma limitação importante. As justificativas para tal se pautaram no diferente nível de conhecimento possivelmente existente ${ }^{3}$, por serem voluntários ${ }^{14}$, por serem médicos do pronto socorro ${ }^{7}$, ou profissionais de uma única localidade ${ }^{2,3,7,20}$. Quatro estudos mencionaram a falta de evidência científica do método como uma limitação $0^{2,3,13,20}$. Um artigo dissertou que as recomendações de consenso, apesar de serem de especialistas, não representaram uma solução suficiente ${ }^{13,23}$ ou não se aplicaram em todos os pacientes ${ }^{16}$. Um artigo afirmou que a incapacidade de realizar trocas de ideias entre os participantes também consiste em limitações da técnica ${ }^{3}$.

As demais limitações foram citadas uma única vez em cada artigo, foram elas: os casos identificados diferiram do diagnóstico médico ${ }^{24}$, a técnica Delphi não representou uma solução suficiente para a base de saúde, ${ }^{13}$ o método não permitiu comparar referências e não foram coletados dados que poderiam influenciar nas decisões, ${ }^{7}$ pode ter ocorrido erro de transcrição ou de percepção das respostas, ${ }^{21}$ a decisão arbitrária do percentual para se caracterizar consenso $^{16}$, perda de seguimento de especialistas ${ }^{18} \mathrm{e}$ pacientes $^{26}$, falta de dados para cálculo ${ }^{26}$, não abordagem de questões políticas e de $\operatorname{custos}^{19}$, a eficácia depende da implementação adequada do protocolo ${ }^{17}$, o feedback aos participantes podem ter influenciado as decisões ${ }^{23}$, a perspectiva foi embasada somente em médicos ${ }^{22}$ e a restrição de tempo para a execução das etapas do método Delphi ${ }^{3}$.

Os resultados da análise dos artigos selecionados para compor a revisão sistemática estão descritos na Tabela 2. 


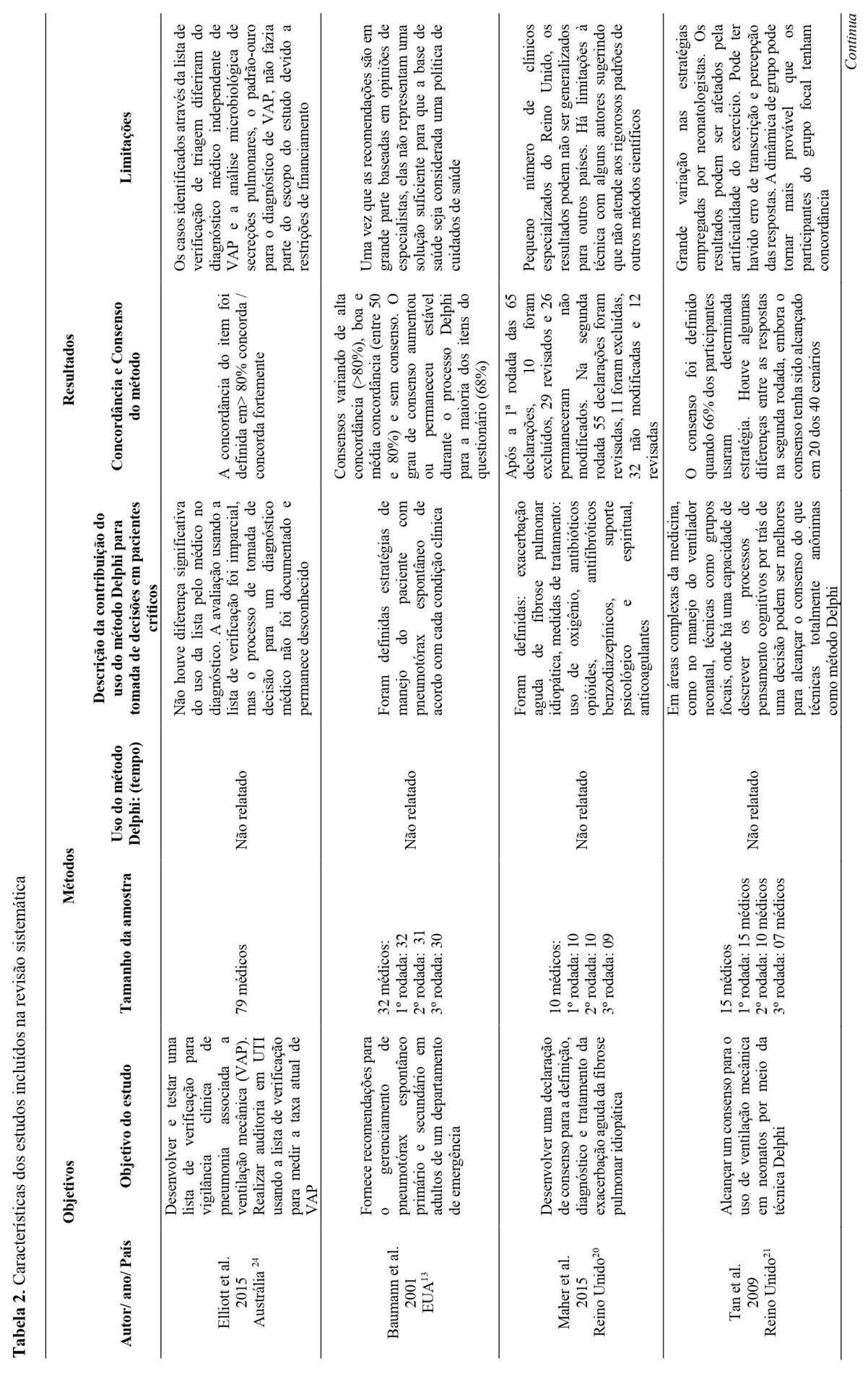




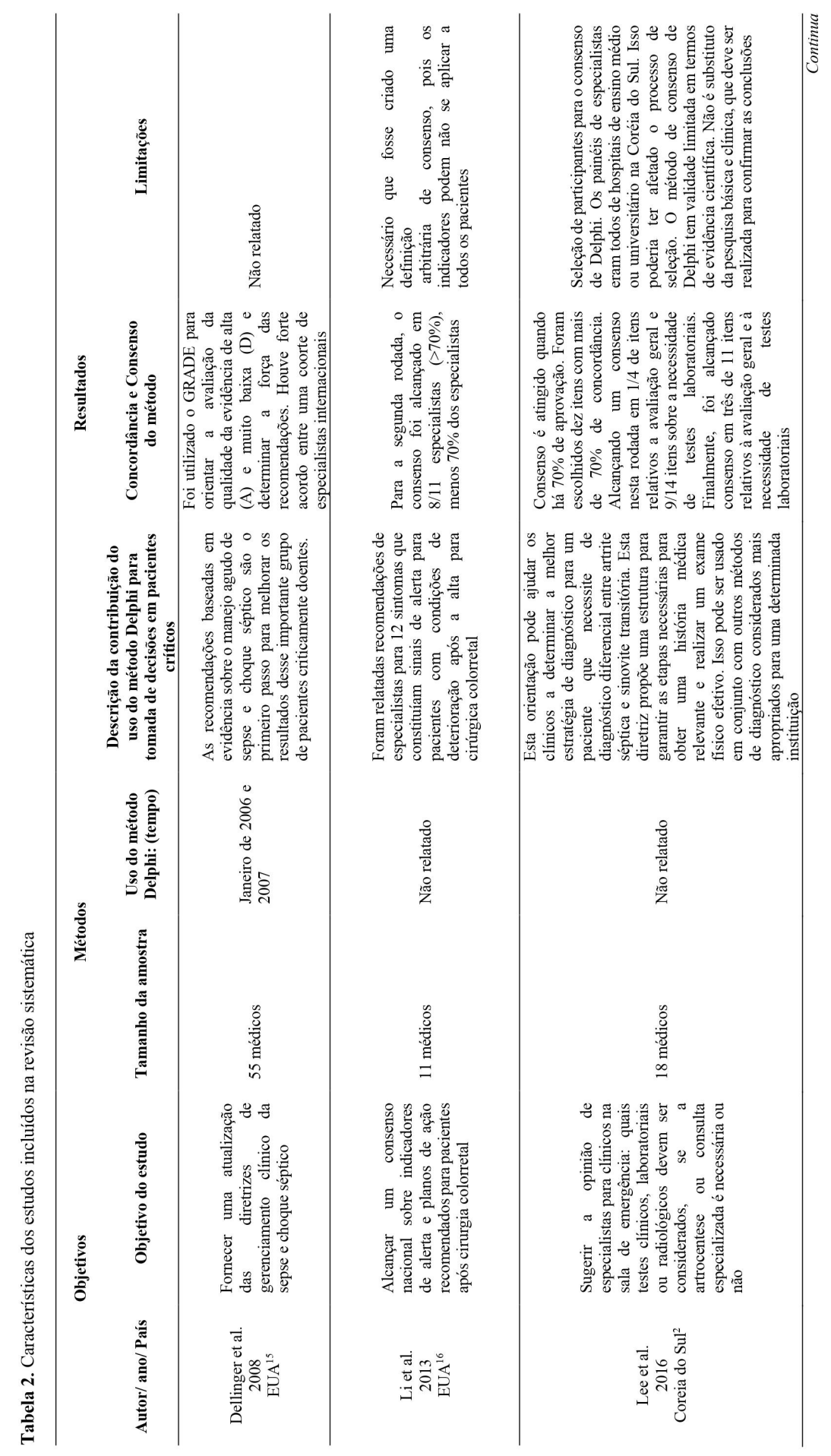




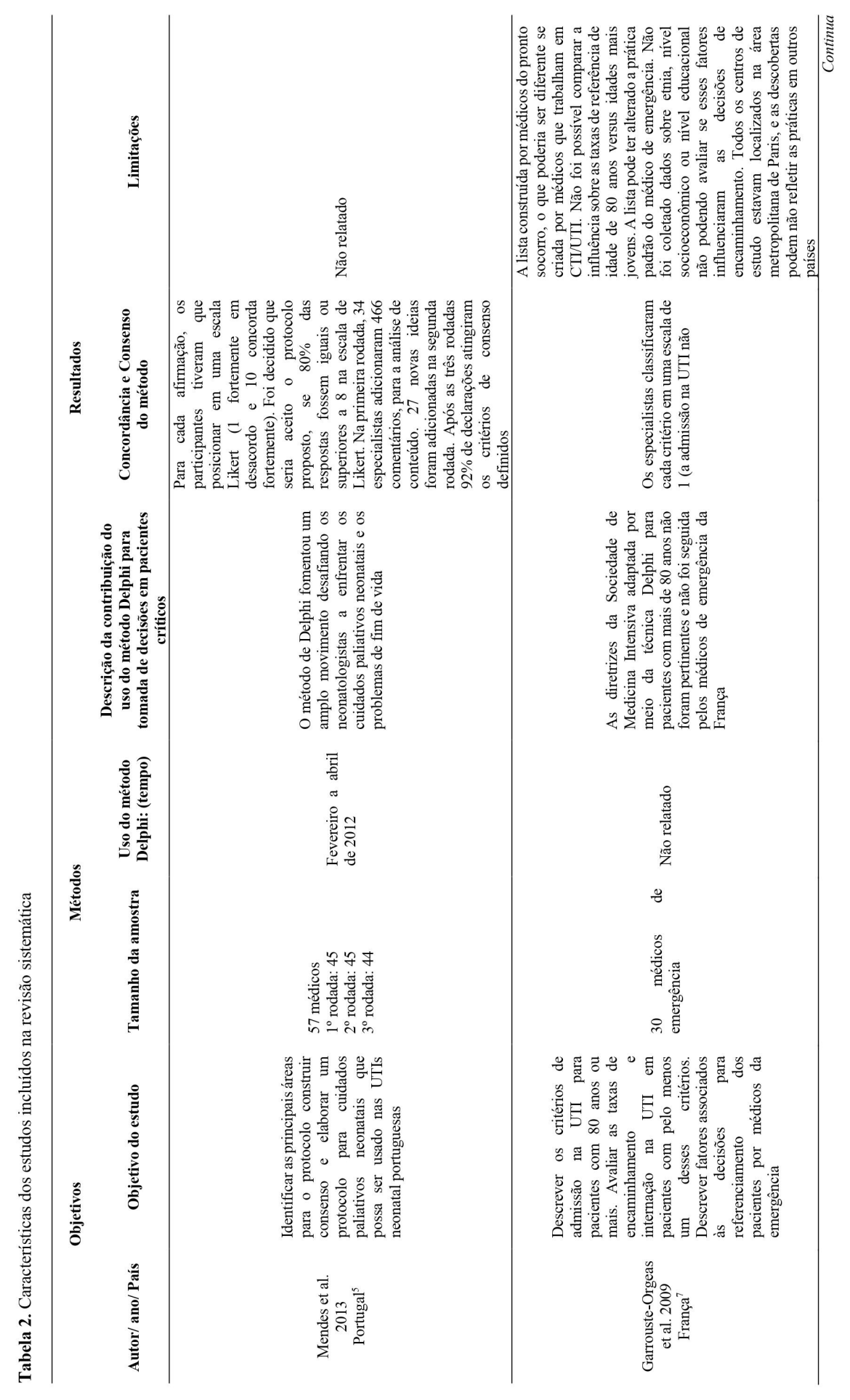




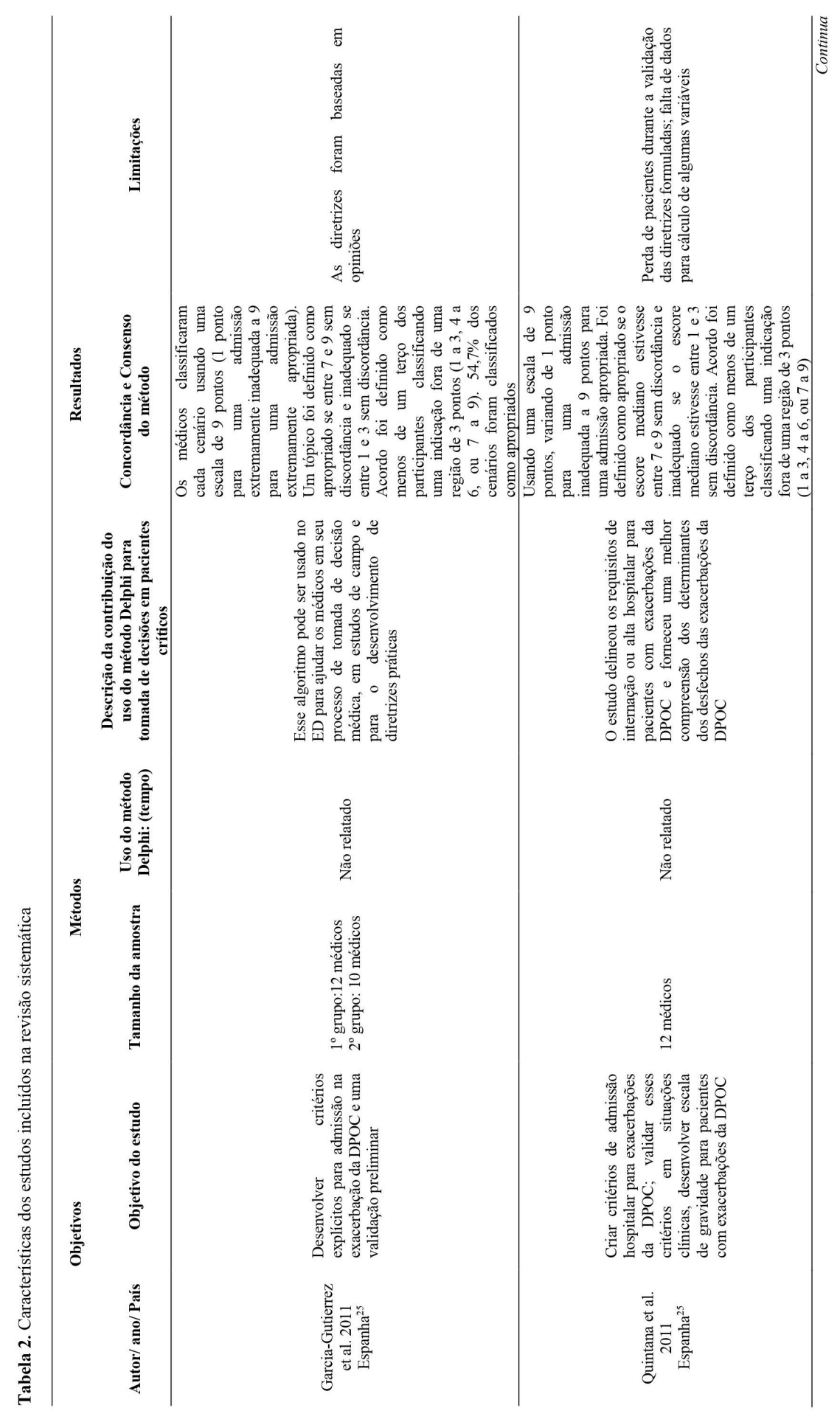




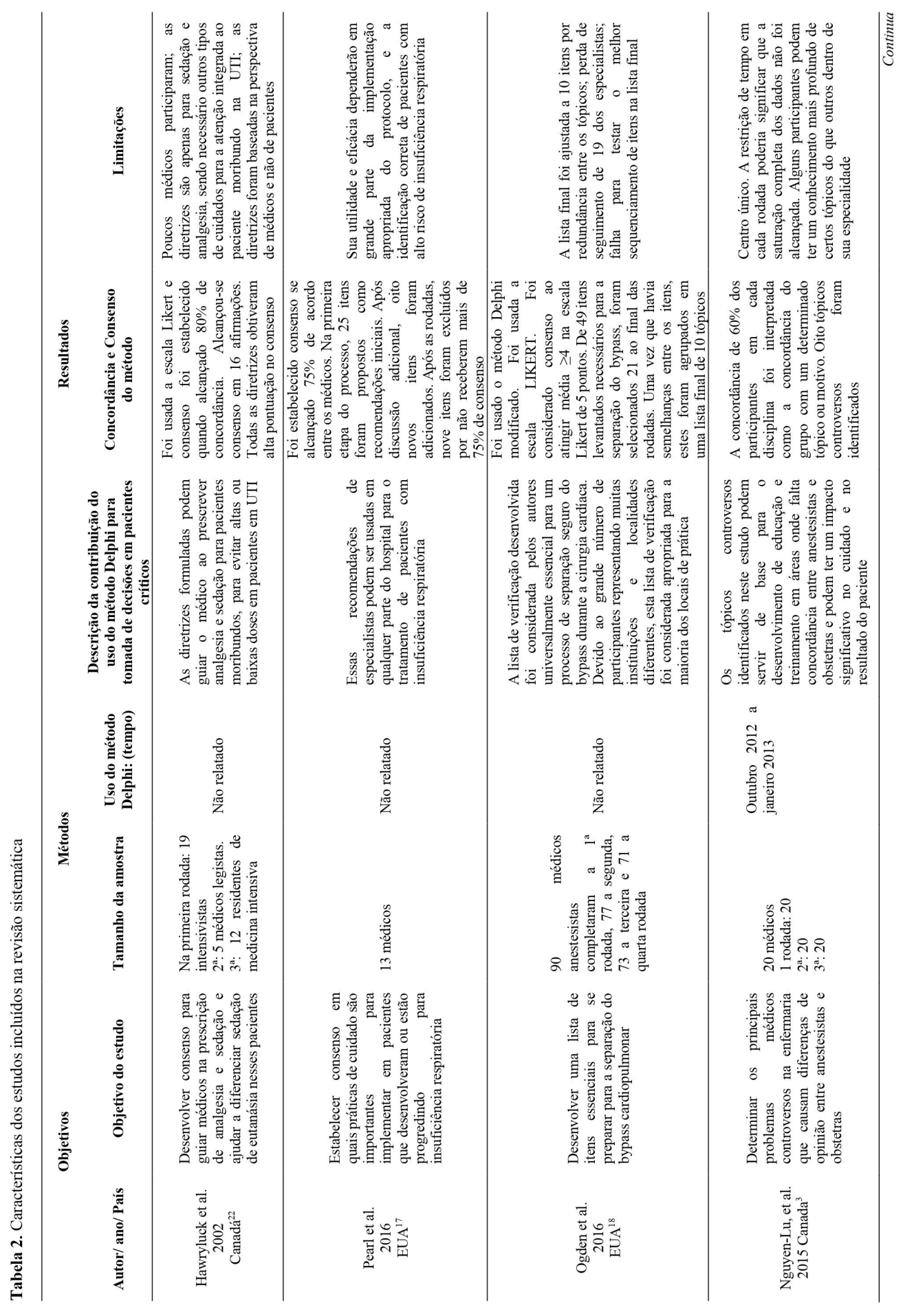




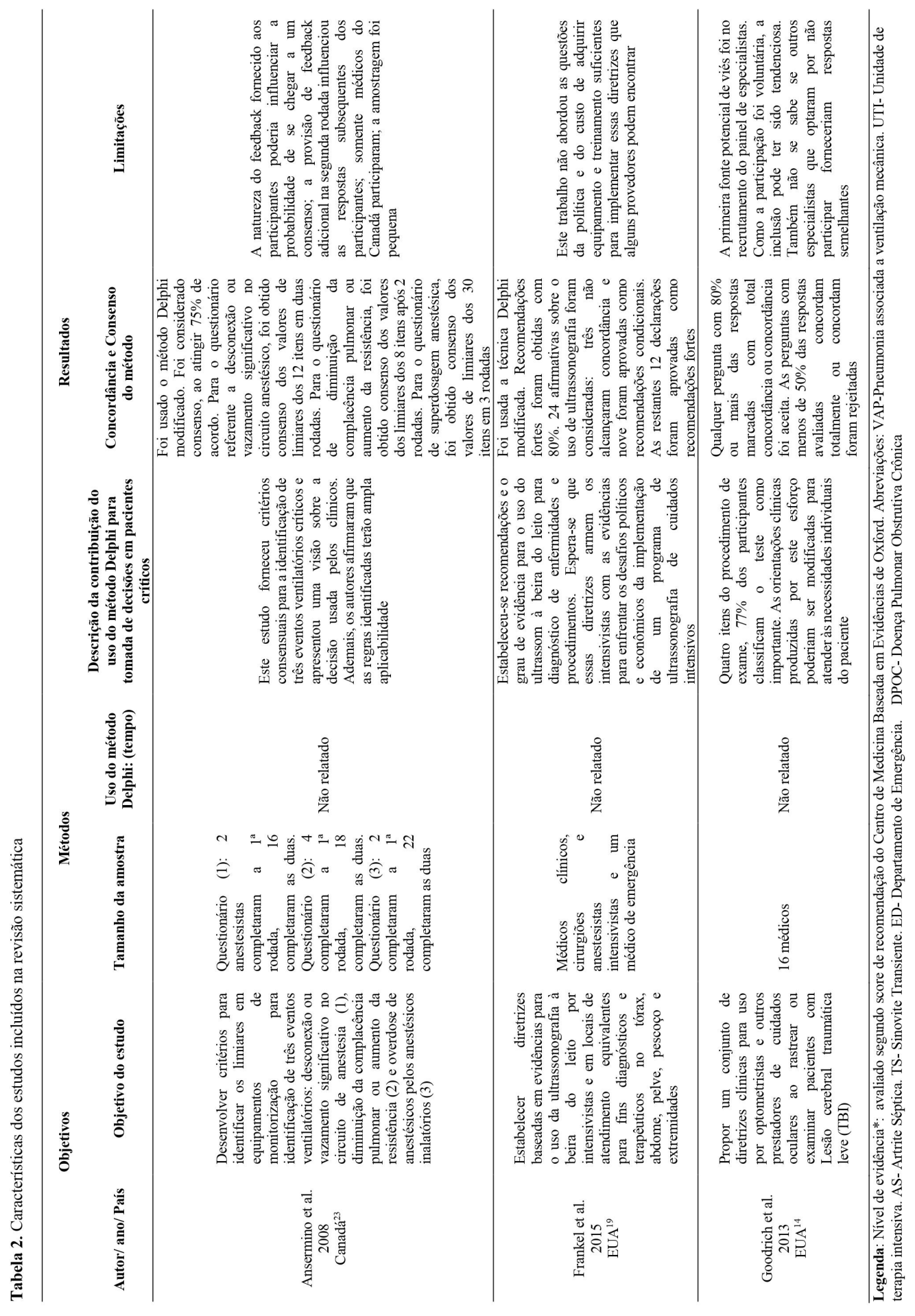




\section{DISCUSSÃO}

O uso da técnica Delphi para tomada de decisões em pacientes críticos contribuiu efetivamente para solucionar empasses no âmbito médico em 15 dos 18 artigos selecionados nesta revisão. As contribuições da técnica Delphi analisadas nos estudos selecionados foram realizadas em áreas de estratégias de manejo, tratamento medicamentoso, recomendações para sintomas específicos, uso de equipamentos, diretrizes para gerenciar e diagnosticar pacientes, consenso para testes e exames e identificação de tópicos de ideias controversas e seus motivos subjacentes ${ }^{2,3,5,13-20,22-26}$.

Deste modo, este estudo analisou a contribuição da técnica Delphi para a condução de pacientes críticos pelos médicos. Contudo, somente três trabalhos mencionaram os resultados finais obtidos com a aplicação das diretrizes estabelecidas: Garrouste-Orgeas ${ }^{7}$, Elliot, et al. ${ }^{24} \mathrm{e}$ Quintana ${ }^{26}$. Esse fato limita a avaliação dos resultados na sobrevida desses pacientes.

Em dez dos 18 artigos publicados de 2001 a 2016, a análise mostrou o uso de uma técnica Delphi modificada. As alterações na aplicação do método citadas consistem em encontros face a face ${ }^{2,15,25,26}$ ou em uma agenda da primeira rodada determinada pelo moderado ${ }^{21} \mathrm{e}$ não pelas respostas da primeira rodada, como na técnica clássica. Os trabalhos de Elliot ${ }^{24}$, Goodrich ${ }^{14}$, Pearl ${ }^{17}$, Ogden ${ }^{18}$, Frankel ${ }^{19}$ e Arsemino $^{23}$ relataram o uso de um método com modificações, contudo não descreveram as mudanças efetuadas. Nestes casos, o método Delphi, descrito de forma incompleta, pode afetar a qualidade geral do consenso final já que as decisões tomadas provavelmente não obterão o nível de credibilidade necessário para a adoção na prática médica. Nos demais trabalhos, a técnica Delphi foi realizada de acordo com a ideia original.

Nove dos 18 trabalhos avaliados não apresentaram desligamento dos médicos participantes no decorrer da aplicação da técnica ${ }^{2,3,7,15-17,19,25,26}$, no restante, a média elevada da porcentagem de permanência de $71,99 \%$ dos participantes no decorrer do processo contribuiu para a confiabilidade das decisões de consenso, além de consolidar a estruturação no método. Ademais, entre todos os trabalhos que compõem essa revisão, dez deles usaram como taxa mínima citada estabelecida para consenso maior que $70 \%$ que consiste em valores já utilizados na literatura como em Harmsen ${ }^{27}$, que, portanto, garantem uma estruturação válida para o método.

Dezesseis dos 18 artigos que compõe essa revisão sistemática citaram as principais limitações encontradas durante a execução do método Delphi ${ }^{2,3,7,13,14,16-26}$. Dentre as limitações apresentadas, as mais recorrentes foram a seleção dos especialistas e a falta de evidência científica do método. Dessa forma, este presente trabalho foi importante como fonte primária de reconhecimento e agrupamento das principais restrições que podem ocorrer durante o processo. No entanto, novos estudos são necessários para validar mecanismos que possam solucionar essas questões.

Esta é a primeira revisão sistemática brasileira que elucida o uso da técnica Delphi para tomada de decisões em pacientes críticos. Os pontos fortes do estudo incluem a recuperação de estudos publicados durante um período de 15 anos (2001-2016) e a análise da contribuição do método para decisões médicas em situações críticas. No entanto, este estudo tem limitações. Não existe consenso sobre como avaliar a aplicabilidade de um procedimento Delphi. Consequentemente, em três dos trabalhos analisados o método não se mostrou efetivo ${ }^{7,21,24}$. Além disso, é difícil avaliar com precisão a efetividade do método, já que não há uma porcentagem mínima e ideal para se chegar a um consenso definida na literatura. Finalmente, não foi possível encontrar ensaios clínicos com o uso da técnica Delphi, uma vez que o tempo necessário para se chegar ao consenso pode ser considerado relativamente extenso para um paciente em situação crítica que necessite de intervenção precoce e rápida. Em três trabalhos ${ }^{3,5,15}$, o período necessário para se completar as rodadas variou entre três meses a um ano.

\section{CONCLUSÃo}

Este estudo mostra a aplicabilidade da técnica Delphi para manejo de pacientes críticos, tratamento medicamentoso, recomendações para sintomas específicos, diretrizes para gerenciar e diagnosticar pacientes, utilização de equipamentos, consenso para testes e exames e identificação de tópicos de ideias controversas e seus motivos subjacentes em situações onde não há consenso na literatura médica. Foi demonstrado que em 15 artigos selecionados, a técnica contribuiu para a formulação de diretrizes médicas.

Agradecimentos: Agradecemos a Universidade de Itaúna e Universidade Federal de São João del-Rei pelo apoio à realização deste trabalho. O presente trabalho foi realizado com apoio da Coordenação de Aperfeiçoamento de Pessoal de Nível Superior - Brasil (CAPES) - Código de Financiamento 001 e da Fundação de Amparo à Pesquisa do Estado de Minas Gerais (FAPEMIG).

Participação dos autores: $S a$ ntos $A P F B$ - contribuiu substancialmente para a concepção, coleta de dados, análise e interpretação dos dados; Andrade JF - contribuiu substancialmente para a concepção, coleta de dados, análise e interpretação dos dados; Alves GCS contribuiu substancialmente para a concepção, coleta de dados, análise e interpretação dos dados, discussão dos resultados e revisão crítica do artigo no que diz respeito ao conteúdo técnico; Silva SD - contribuiu substancialmente para a concepção, coleta de dados, análise e interpretação dos dados; Sanches $C$ - contribuiu para a elaboração do trabalho, supervisão da coleta de dados, orientação e análises dos dados, discussão dos resultados e revisão crítica do artigo no que diz respeito ao conteúdo técnico; Chequer FMD - contribuiu para a elaboração do trabalho, supervisão da coleta de dados, orientação e análises dos dados, discussão dos resultados e revisão crítica do artigo no que diz respeito ao conteúdo técnico. 


\section{REFERÊNCIAS}

1. Habibi A, Sarafrazi A, Izadyar S. Delphi Technique Theoretical Framework in Qualitative Research. Int J Eng Sci. 2014;3(4):8-13. Available from: http://theijes.com/papers/ v3-i4/Version-4/B03404008013.pdf.

2. Lee JH, Park MS, Kwon H, Chung CY, Lee KM, Kim YJ, et al. A guideline for differential diagnosis between septic arthritis and transient synovitis in the emergency department: a Delphi survey. Am J Emerg Med. 2016;34(8):1631-6. doi: 10.1016/j.ajem.2016.06.006.

3. Nguyen-Lu N, Downey K, Carvalho JC. Controversy between anesthesiologists and obstetricians on the labour ward: the Delphi method is used as a consensus-building technique. Can J Anesth. 2015;62(3):271-7. doi: 10.1007/s12630-0140294-7.

4. Wassenaar A, Van den Boogaard M, Underpin-Icu Study Group, Schoonhoven L, Pickkers P. Determination of the feasibility of a multicomponent intervention program to prevent delirium in the Intensive Care Unit: a modified RAND Delphi study. Aust Crit Care. 2017;30(6):321-7. doi: 10.1016/j.aucc.2016.12.004.

5. Mendes JC, Silva LJ. Neonatal palliative care: developing consensus among neonatologists using the Delphi Technique in Portugal. Adv Neonatal Care. 2013;13(6):408-14. doi: 10.1097/ANC.0000000000000037.

6. Strang JF, Meagher H, Kenworthy L, de Vries AC, Menvielle E, Leibowitz $\mathrm{S}$, et al. Initial Clinical Guidelines for CoOccurring Autism Spectrum Disorder and Gender Dysphoria or Incongruence in Adolescents. J Clin Child Adolesc Psychol. 2018;47(1):105-15. doi: 10.1080/15374416.2016.1228462.

7. Garrouste-Orgeas M, Boumendil A, Pateron D, Aergerter P, Somme D, Simon T, et al. Selection of intensive care unit admission criteria for patients aged 80 years and over and compliance of emergency and intensive care unit physicians with the selected criteria: An observational, multicenter, prospective study. Crit Care Med. 2009;37(11):2919-28. doi: 10.1097/ccm.0b013e3181b019f0.

8. Sousa AS, Cunha CP, Magalhães LC, Kaiser SE, Saraiva JK. Posicionamentos, diretrizes e normatizações. Veículo de auxílio à prática médica. Arq Bras Cardiol. 2017;109(4):3689. doi:10.5935/abc. 20170133 .

9. Moher D, Liberati A, Tetzlaff J, Altman DG, PRISMA Group. Preferred reporting items for systematic reviews and meta- analyses: the PRISMA statement. PLoS Med. 2009;6(7):e1000097. doi: 10.1371/journal.pmed.1000097.

10. Galvão TF, Pereira MG. Revisões sistemáticas da literatura: passos para sua elaboração. Epidemiol Serv Saúde. 2014;23(1):183-4. doi: 10.5123/S1679-49742014000100018.

11. Phillips B, Ball C, Sackett D, Badenoch D, Straus S, Haynes B, et al. Oxford Centre for Evidence-based Medicine - Levels of evidence (March 2009). Oxford: Centre for EvidenceBased Medicine (CEBM); 2009 [cited 2018 Feb 18]. Available from: http://www.cebm.net/index.aspx?o=1025.

12. Landis JR, Koch GG. The measurement of observer agreement for categorical data. Biometrics. 1977;33(1):15974. doi: $10.2307 / 2529310$.
13. Baumann MH, Strange C, Heffner JE, Light R, Kirby TJ, Klein J, et al. Management of Spontaneous Pneumothorax: an American College of Chest Physicians Delphi Consensus Statement. Chest. 2001;119(2):590-602. doi: 10.1378/ chest.119.2.590.

14. Goodrich GL, Martinsen GL, Flyg HM, Kirby J, Asch $\mathrm{SM}$, Brahm KD, et al. Development of a mild traumatic brain injury-specific vision screening protocol: a Delphi study. J Rehabil Res Dev. 2013;50(6):757-68. doi: 10.1682/ JRRD.2012.10.0184.

15. Dellinger RP, Levy MM, Carlet JM, Bion J, Parker MM, Jaeschke R, et al. Surviving Sepsis Campaign: International guidelines for management of severe sepsis and septic shock: 2008. Intens Care Med. 2008;34(1):17-60. doi: 10.1007/ s00134-007-0934-2.

16. Li LT, Mills WL, Gutierrez AM, Herman LI, Berger DH, Naik AD. A patient-centered early warning system to prevent readmission after colorectal surgery: a national consensus using the Delphi method. J Am Coll Surg. 2013;216(2):210-6. doi: 10.1016/j.jamcollsurg.2012.10.011.

17. Pearl JS, Gajic O, Dong Y, Herasevich V, Gong MN. Creation of the Prevention of Organ Failure Checklist. A Multidisciplinary Approach Using the Modified Delphi Technique. Ann Am Thorac Soc. 2016;13(6):910-6. doi: 10.1513/AnnalsATS.201509-626BC.

18. Ogden SR, Culp WC, Villamaria FJ, Ball TR. Developing a checklist: Consensus Via a Modified Delphi Technique. J Cardiothorac Vasc Anesth. 2016;30(4):855-8. doi: 10.1053/j. jvca.2016.02.022.

19. Frankel HL, Kirkpatrick AW, Elbarbary M, Blaivas M, Desai H, Evans D, et al. Guidelines for the Appropriate Use of Bedside General and Cardiac Ultrasonography in the Evaluation of Critically Ill Patients-Part I: General Ultrasonography. Crit Care Med. 2015;43(11):2479-502. doi: 10.1097/CCM.0000000000001216

20. Maher TM, Whyte MK, Hoyles RK, Parfrey H, Ochiai Y, Mathieson N, et al. Development of a Consensus Statement for the Definition, Diagnosis, and Treatment of Acute Exacerbations of Idiopathic Pulmonary Fibrosis Using the Delphi Technique. Adv Ther. 2015;32(10):929-43. doi: 10.1007/s12325-015-0249-6.

21. Tan K, Baxter G, Newell S, Smye S, Dear P, Brownlee K, et al. Knowledge elicitation for validation of a neonatal ventilation expert system utilising modified Delphi and focus group techniques. Int J Hum Comput Stud. 2009;68(6):344-54. doi: 10.1016/j.ijhcs.2009.08.003.

22. Hawryluck LA, Harvey WR, Lemieux-Charles L, Singer PA. Consensus guidelines on analgesia and sedation in dying intensive care unit patients. BMC Med Ethics. 2002;12;3:E3. doi: 10.1186/1472-6939-3-3.

23. Ansermino JM, Dosani M, Amari E, Choi PT, Schwarz SW. Defining rules for the identification of critical ventilatory events. Can J Anesth. 2008;55(10):702-714. doi: 10.1007/ bf03017747

24. Elliott D, Elliott R, Burrell A, Harrigan P, Murgo M, Rolls $\mathrm{K}$, et al. Incidence of ventilator-associated pneumonia in Australasian intensive care units: use of a consensus- 
developed clinical surveillance checklist in a multisite prospective audit. BMJ Open. 2015;5(10):e008924. doi: 10.1136/bmjopen-2015-008924.

25. Garcia-Gutierrez S, Quintana JM, Aguirre U, Esteban C, Bilbao A, Escobar A, et al. Explicit criteria for hospital admission in exacerbations of chronic obstructive pulmonary disease. Int J Tuberc Lung Dis. 2011;15(5):680-6. doi: 10.5588/ijtld.10.0408.

26. Quintana JM, Esteban C, Barrio I, Garcia-Gutierrez S, Gonzalez N, Arostegui I, et al. The IRYSS-COPD appropriateness study: objectives, methodology, and description of the prospective cohort. BMC Health Serv Res. 2011;11:322. doi: 10.1186/1472-6963-11-322.

27. Harmsen AM, Geeraedts LM, Giannakopoulos GF, Terra M, Christiaans HM, Mokkink LB, et al. Protocol of the DENIM study: a Delphi-procedure on the identification of trauma patients in need of care by physician-staffed Mobile Medical Teams in the Netherlands. Scand J Trauma Resusc Emerg Med. 2015;23:15. doi: 10.1186/s13049-015-0089-z.

Recebido: 04/08/2019

Aceito: 17/03/20 